\title{
Magnetic fields in classical Be stars: results of our long-term program with FORS1 at the VLT
}

\author{
Ruslan V. Yudin ${ }^{1}$, Swetlana Hubrig ${ }^{2}$, Michail A. Pogodin ${ }^{1}$ and \\ Markus Schoeller ${ }^{3}$ \\ ${ }^{1}$ Central Astronomical Observatory of the Russian Academy of Sciences at Pulkovo, \\ Saint-Petersburg, Russia, email: ruslan61@gao.spb.ru \\ ${ }^{2}$ Astrophysical Institute Potsdam, Germany \\ ${ }^{3}$ European Southern Observatory, Garching, Germany
}

\begin{abstract}
We report the results of our search for magnetic fields in a representative sample of classical Be stars carried out during 2006-2008 using low-resolution spectropolarimetry with FORS1 at the VLT. Among the 28 classical Be stars studied, detections of a magnetic field were achieved in seven stars (i.e. $\sim 25 \%$ ). The detected magnetic fields are rather weak, not stronger than $\sim 150 \mathrm{G}$. Among the Be stars studied with time series, one Be star, $\lambda$ Eri, displays cyclic variability of the magnetic field with a period of $21.12 \mathrm{~min}$.
\end{abstract}

Keywords. stars: emission-line, Be, magnetic fields

\section{Introduction}

A number of physical processes in classical Be stars (e.g., angular momentum transfer to a CS disk, channelling stellar wind matter, accumulation of material in an equatorial disk, etc.) are more easily explainable if magnetic fields are invoked (e.g. Brown et al. 2004; Cassinelli et al. 2002). Maheswaran (2003, 2005) developed the Magnetic Rotator Wind Disk model, in which Keplerian disks may be formed by magnetic fields of the order of a few tens of Gauss. Very recently, Maheswaran \& Cassinelli (2009) obtained solutions for the structure and evolution of a protodisk region, i.e. the disk region that is initially formed when wind material is channelled by dipole-type magnetic fields towards the equatorial plane, showing that magnetorotational instability may assist in the formation of a quasisteady disk. According to their calculations, magnetic fields of the order of a few tens of Gauss will be able to channel wind flow into a protodisk region. Due to the high rotation of Be stars and the presence of strong Balmer emission lines, magnetic field measurements are difficult. We report here the results of our search for magnetic fields in a representative sample of classical Be stars carried out during four observing runs in the years 2005-2008 using low-resolution spectropolarimetry with FORS 1 at the VLT.

\section{Results and Discussion}

During our runs with FORS1 using low-resolution (R 2000 and 4000) spectropolarimetric data we studied 28 Be stars in the range of spectral classes from B9 to O8. Among this sample the detections of a magnetic field were achieved in 8 stars, most of them just occasionally on single nights (Hubrig et al. 2007; 2009a; 2009b). The magnetic fields of Be stars appear to be very weak, generally of the order of $100 \mathrm{G}$ and less. Significant polarization features have been revealed in CS components of the Ca II K\&H lines in the spectra of 5 Be stars. No doubt that these lines are of circumstellar (CS) origin. These 
features are likely to be formed in the equatorial gaseous disks surrounding the stars. Besides, a clear Stokes V signature is observed in a redshifted absorption component of the $\mathrm{H}_{\beta}$ line profile in the spectrum of HD58011. Profiles of such a type are indicators of the presence of matter infall from the CS disk onto the star. Therefore, we can assume that the infalling flow in the CS envelope of HD58011 is magnetized. Note that the magnetic field detection in Be stars is independent of their spectral classes and was achieved for stars of spectral types from B9 to O8. In a framework of our observing programs we also carried out time resolved magnetic field measurements of nine classical Be stars. We were able to obtain during one hour 20-30 consecutive measurements for the stars depending on its brightness.

In the obtained amplitude spectra a $2.4 \sigma$ peak corresponding to a period of $21.12 \mathrm{~min}$ was detected in the data set of measurements carried out using hydrogen lines in the star $\lambda$ Eri in August 2006. This peak appears at a $2.2 \sigma$ in the data set of measurements carried out using the whole spectrum. Note, however, that our observations obtained 16 months later on two consecutive nights on 2007 November 27 and 28 over few hours did not reveal any significant periodicity in any of the data sets. Other Be stars: QY Car, $\delta$ Cen, a Ara, and $\epsilon$ Tuc show weak signals in the Fourier transforms of our data sets, corresponding to periods of $21.86 \mathrm{~min}, 27.74 \mathrm{~min}, 9.37 \mathrm{~min}$, and $4.27 \mathrm{~min}$, respectively.

Actually, we found that five stars out of nine from our program with time series show periodic phase variations of magnetic fields with short periods (minutes, tens minutes). Since the topology of the magnetic field is not known, it is difficult to estimate the impact of non-radial pulsations causing strong line asymmetries on our measurements. It is quite possible that lines of different elements behave differently with respect to their pulsation amplitudes and shapes of the line profiles. Nevertheless, non-radial pulsations can be responsible for a periodic spectral and photometric variability observed on short-term scales (less than 1 day).

\section{Conclusions}

Our search for magnetic fields in Be stars revealed that while their magnetic fields are rather weak, fields of the order of $100 \mathrm{G}$ and less are not rare ( 7 Be stars out of 28 or $1 / 4$ ). Since a large fraction of stars in our sample was observed only once, a non-detection of their magnetic field may be explained by temporal variability of their magnetic fields. A cyclical variability with short periods (minutes, tens of minutes) was detected in 5 stars out of nine with time series. Observations of Be stars at different stages of their phase transition "Be $\rightarrow$ Be-shell $\rightarrow$ normal B" would be very useful for understanding of the role of magnetic fields to Be-phenomenon. It is beyond doubt that magnetic fields are present in Be stars, but they are not strong !

\section{References}

Brown, J. C., Telfer, D., Li, Q., Hanuschik, R. et al. 2004, MNRAS 352, 1061

Cassinelli, J. P., Brown, J. C., Maheswaran, M., Miller, N. A. et al. 2002, ApJ 578, 951

Hubrig, S., Yudin, R. V., Pogodin, M., Schöller, M. et al. 2007, AN 328, 1133

Hubrig, S., Schöller, M., Savanov, I., Yudin, R. V. et al. 2009a, AN 330, 708

Hubrig, S., Schöller, M., Briquet, M., De Cat, P. et al. 2009b, The Messenger 135, 21

Maheswaran, M. 2003, ApJ 592, 1156

Maheswaran, M. 2005, in: R. Ignace \& K. G. Gayley (eds.), The Nature and Evolution of Disks Around Hot Stars, ASP-CS 337, p. 259

Maheswaran, M. \& Cassinelli, J. P. 2009, MNRAS 394, 415 